USM-TH-147

\title{
Static potential in a topologically massive Born-Infeld theory
}

\author{
Patricio Gaete * \\ Departamento de Física, Universidad Técnica F. Santa María, Valparaíso, Chile
}

\begin{abstract}
For a (2+1)-dimensional topologically massive Born-Infeld theory, we compute the interaction potential within the structure of the gauge-invariant but path-dependent variables formalism. The result is equivalent to that of $Q E D_{3}$ with a Thirring interaction term among fermions, in the short distance regime.
\end{abstract}

PACS number(s): 11.10.Ef, 11.10.Kk

\section{INTRODUCTION}

Topologically massive gauge theories in three-dimensional spacetime have been studied by various authors in the last few years [1-3]. An example of such a class of theories is the Maxwell-Chern-Simons theory, which is endowed with interesting features such as a massive gauge field and physical excitations with fractional statistics (anyons) which interpolate between bosons and fermions. In addition, they are interesting because of its connection to the high-temperature limit of four-dimensional theories [4,5] as well as for their applications to condensed matter physics [6].

We further observe that recently a great deal of attention has been devoted to the study of non-linear electrodynamics (Born-Infeld theory) due to its arising naturally in D-brane physics $[7,8]$. Let us also recall here that Born and Infeld [9] suggested to modify Maxwell's

*E-mail: patricio.gaete@fis.utfsm.cl 
electromagnetism with the goal of avoiding divergences such as the infinite self-energy of a point charge.

On the other hand, the study of duality symmetry in gauge theories has also attracted considerable attention in order to provide an equivalent description of physical phenomena by distinct theories. As is well known, duality refers to an equivalence relation between two or more quantum field theories whose corresponding classical theories are different. An interesting and illustrative example on this subject arises when one considers three dimensions of spacetime, where it was shown in Refs. $[1,10,11]$ that the self-dual and Maxwell-ChernSimons theories are identical as quantum theories. Within this context a $(2+1)$-dimensional topologically massive Born-Infeld theory has been studied in Ref. [12]. However, in this Letter we wish to further elaborate on the physical content of this theory. To this end we will compute the lowest order modification of the static potential due to the presence of the Born-Infeld term. In fact, we will show that the static potential for the topologically massive Born-Infeld theory agrees with that of the Maxwell-Chern-Simons theory with a Thirring interaction term among fermions, in the short distance regime. In this way we establish a new connection between both theories, in the hope that this will be helpful to understand better effective gauge theories in $(2+1)$ dimensions. Our calculation is based on the gauge-invariant but path-dependent variables formalism. According to this formalism, the interaction potential between two static charges is obtained once a judicious identification of the physical degrees of freedom is made [13]. This methodology, in our view, is of interest both for its simplicity and physical content.

\section{INTERACTION ENERGY}

As already stated, our objective is to compute explicitly the interaction energy between static pointlike sources for topologically massive Born-Infeld theory. To this end we shall first carry out its Hamiltonian analysis. The starting point is the Lagrangian [12]:

$$
\mathcal{L}=\beta^{2}\left\{1-\sqrt{1+\frac{1}{2 \beta^{2}} F_{\mu \nu} F^{\mu \nu}}\right\}+\frac{\theta}{4} \varepsilon^{\mu \nu \lambda} A_{\mu} F_{\nu \lambda}-A_{0} J^{0},
$$


where $J^{0}$ is the external current. The parameter $\beta$ measures the nonlinearity of the theory and in the limit $\beta \rightarrow \infty$ the Lagrangian (1) reduces to the Maxwell-Chern-Simons theory. In order to handle the square root in the Lagrangian (1), we introduce an auxiliary field $v$, such that its equation of motion gives back the original theory [7]. This allows us to rewrite the Lagrangian (1) in the form

$$
\mathcal{L}=\beta^{2}\left\{1-\frac{v}{2}\left(1+\frac{1}{2 \beta^{2}} F_{\mu \nu} F^{\mu \nu}\right)-\frac{1}{2 v}\right\}+\frac{\theta}{4} \varepsilon^{\mu \nu \lambda} A_{\mu} F_{\nu \lambda}-A_{0} J^{0}
$$

In order to derive the canonical Hamiltonian, we note that the canonical momenta are $\Pi^{\mu}=$ $v F^{\mu 0}+\frac{\theta}{2} \varepsilon^{0 \mu \nu} A_{\nu}$. This yields the usual primary constraint $\Pi^{0}=0$ and $p \equiv \frac{\partial \mathcal{L}}{\partial \dot{v}}=0$. Standard techniques for constrained systems then lead to the following canonical Hamiltonian

$$
\begin{array}{r}
H_{C}=\int d^{2} x\left\{-\frac{1}{2 v}\left(\Pi_{i} \Pi^{i}+\theta \varepsilon^{i j} A_{i} \Pi_{j}\right.\right. \\
\left.\left.+\frac{\theta^{2}}{2} A_{i} A^{i}\right)+\frac{v}{2}\left(\frac{1}{2} F_{i j} F^{i j}+\beta^{2}\right)-\beta^{2}\right\}+ \\
+\int d^{2} x\left\{-A_{0}\left(\partial_{i} \Pi^{i}+\frac{\theta}{2} \varepsilon^{i j} \partial_{i} A_{j}-J^{0}\right)\right\} .
\end{array}
$$

Temporal conservation of the primary constraint $\Pi^{0}$ leads to the secondary constraint $\Gamma_{1}(x) \equiv \partial_{i} \Pi^{i}+\frac{\theta}{2} \varepsilon^{i j} \partial_{i} A_{j}-J^{0}=0$. The consistency condition for the $p$ constraint yields no further constraints and just determines the field $v$,

$$
v=\sqrt{\frac{1-\frac{1}{\beta^{2}}\left(\Pi^{i} \Pi_{i}+\theta \varepsilon^{i j} A_{i} \Pi_{j}+\frac{\theta^{2}}{2} A^{i} A_{i}\right)}{1+\frac{1}{2 \beta^{2}} F^{i j} F_{i j}}},
$$

which will be used to eliminate $v$. The extended Hamiltonian that generates translations in time then reads $H=H_{C}+\int d x\left(c_{0}(x) \Pi_{0}(x)+c_{1}(x) \Gamma_{1}(x)\right)$, where $c_{0}(x)$ and $c_{1}(x)$ are the Lagrange multipliers. Since $\Pi_{0}=0$ for all time and $\dot{A}_{0}(x)=\left[A_{0}(x), H\right]=c_{0}(x)$, which is completely arbitrary, we discard $A_{0}(x)$ and $\Pi_{0}(x)$ because they add nothing to the description of the system. As a result, the Hamiltonian becomes

$$
H=\int d^{2} x\left\{\beta^{2} \sqrt{\left(1+\frac{B^{2}}{\beta^{2}}\right)\left(1+\frac{\mathbf{D}^{2}}{\beta^{2}}\right)}-\beta^{2}-c^{\prime}(x)\left(\partial_{i} \Pi^{i}+\theta \varepsilon^{i j} \partial_{i} A_{j}-J^{0}\right)\right\},
$$

where $c^{\prime}(x)=c_{1}(x)-A_{0}(x), B^{2}=\frac{1}{2} F_{i j} F^{i j}$ and $\mathbf{D}^{2}=-\left(\Pi_{i} \Pi^{i}+\theta \varepsilon^{i j} A_{i} \Pi_{j}+\frac{\theta^{2}}{2} A_{i} A^{i}\right)$. 
Along with the first class constraint $\Gamma_{1}(x)$ (Gauss' law) we impose one gauge constraint such that the full set of constraints becomes second class. A convenient choice is found to be

$$
\Omega_{2}(x) \equiv \int_{C_{\xi x}} d z^{\nu} A_{\nu}(z)=\int_{0}^{1} d \lambda x^{i} A_{i}(\lambda x)=0,
$$

where $\lambda(0 \leq \lambda \leq 1)$ is the parameter describing the spacelike straight path between the reference points $\xi^{k}$ and $x^{k}$, on a fixed time slice. For simplicity we have assumed the reference point $\xi^{k}=0$. The choice (6) leads to the Poincaré gauge [14]. According to the Dirac method, we arrive at the following nonvanishing Dirac bracket:

$$
\left\{A_{i}(x), \pi^{j}(y)\right\}^{*}=g_{i}^{j} \delta^{(2)}(x-y)-\partial_{i}^{x} \int_{0}^{1} d \lambda x^{j} \delta^{(2)}(\lambda x-y)
$$

Since we are interested in estimating the lowest-order correction to the interaction energy, we will retain only the leading quadratic term in the expression (5). Thus the Hamiltonian may be written as

$$
H=\int d^{2} x\left(\frac{1}{2} \mathbf{D}^{2}+\frac{1}{2} B^{2}\right)
$$

In order to make clear our subsequent work, we now write the Dirac brackets in terms of $B=\varepsilon_{i j} \partial^{i} A^{j}$ and $D^{i}=\Pi^{i}-\frac{\theta}{2} \varepsilon^{i j} A_{j}$ fields, that is,

$$
\begin{gathered}
\left\{D_{i}(x), D_{j}(y)\right\}^{*}=-\theta \varepsilon_{i j} \delta^{(2)}(x-y), \\
\{B(x), B(y)\}^{*}=0, \\
\left\{D_{i}(x), B(y)\right\}^{*}=-\varepsilon_{i j} \partial_{x}^{j} \delta^{(2)}(x-y) .
\end{gathered}
$$

It gives rise to the following equations of motion for $D_{i}$ and $B$ fields:

$$
\begin{gathered}
\dot{D}_{i}(x)=-\theta \varepsilon_{i j} D_{j}(x)+\varepsilon_{i j} \partial_{j} B(x), \\
\dot{B}(x)=-\varepsilon_{i j} \partial_{i} D_{j}(x) .
\end{gathered}
$$


In the same way, we write the Gauss law as:

$$
\partial_{i} D_{L}^{i}+\theta B-J^{0}=0
$$

where $D_{L}^{i}$ refers to the longitudinal part of $D^{i}$. As a consequence of Eqs. (12) and (13), the static fields are given by

$$
\begin{gathered}
B=-\frac{\theta J^{0}}{\nabla^{2}-\theta^{2}}, \\
D_{i}=\frac{1}{\theta} \partial_{i} B
\end{gathered}
$$

where $\nabla^{2}$ is the two-dimensional Laplacian. For $J^{0}(t, \mathbf{x})=e \delta^{(2)}(\mathbf{x}-\mathbf{a})$, expressions (15) and (16) immediately show that

$$
\begin{gathered}
B(x)=\frac{e \theta}{2 \pi} K_{0}(\theta|\mathbf{x}-\mathbf{a}|), \\
D^{i}(x)=-\frac{e \theta}{2 \pi} \frac{|\mathbf{x}-\mathbf{a}|^{i}}{|\mathbf{x}-\mathbf{a}|} K_{1}(\theta|\mathbf{x}-\mathbf{a}|),
\end{gathered}
$$

where $K_{0}$ and $K_{1}$ are modified Bessel functions. However, it follows from the above discussion that the electric field takes the form

$$
E^{i}(x)=\left\{1-\frac{1}{2}\left(\frac{e \theta}{2 \pi \beta}\right)^{2}\left[K_{1}^{2}(\theta|\mathbf{x}-\mathbf{a}|)-K_{0}^{2}(\theta|\mathbf{x}-\mathbf{a}|)\right]\right\} \partial^{i}\left(-\frac{J^{0}}{\nabla^{2}-\theta^{2}}\right) .
$$

We pass now to the calculation of the potential energy for a pair of static pointlike opposite charges at $\mathbf{y}$ and $\mathbf{y}^{\prime}$. The procedure we shall follow is based on the expression

$$
V \equiv e\left(\mathcal{A}_{0}(\mathbf{y})-\mathcal{A}_{0}\left(\mathbf{y}^{\prime}\right)\right)
$$

where the physical scalar potential $\mathcal{A}_{0}$ is expressed in terms of the electric field

$$
\mathcal{A}_{0}(t, \mathbf{x})=\int_{0}^{1} d \lambda x^{i} E_{i}(t, \lambda \mathbf{x})
$$

At this point it is worth to emphasize that Eq.(21) follows from the vector gauge-invariant field [13]: 


$$
\mathcal{A}_{\mu}(x) \equiv A_{\mu}(x)+\partial_{\mu}\left(-\int_{\xi}^{x} d z^{\mu} A_{\mu}(z)\right)
$$

where, as in Eq.(6), the line integral appearing in the above expression is along a spacelike path from the point $\xi$ to $x$, on a fixed time slice. At the same time, it should be noted that the gauge-invariant variables (22) commute with the sole first class constraint (Gauss' law), supporting the fact that these fields are physical variables [15].

Having made these observations and using Eq.(21), we can write immediately the following expression for the physical scalar potential:

$$
\mathcal{A}_{0}(t, \mathbf{x})=\int_{0}^{1} d \lambda\left\{1-\frac{1}{2}\left(\frac{e \theta}{2 \pi \beta}\right)^{2}\left[K_{1}^{2}(\theta|\lambda \mathbf{x}-\mathbf{a}|)-K_{0}^{2}(\theta|\lambda \mathbf{x}-\mathbf{a}|)\right]\right\} x^{i} \partial_{i}^{\lambda \mathbf{x}}\left(-\frac{J^{0}(\lambda \mathbf{x})}{\nabla_{\lambda \mathbf{x}}^{2}-\theta^{2}}\right)
$$

where $J^{0}$ is the external current. Again, for $J^{0}(t, \mathbf{x})=e \delta^{(2)}(\mathbf{x}-\mathbf{a})$, Eq.(23) becomes

$$
\mathcal{A}_{0}(t, \mathbf{x})=\frac{e}{2 \pi}\left(K_{0}(\theta|\mathbf{x}-\mathbf{a}|)-K_{0}(\theta|\mathbf{a}|)\right)-\frac{e^{3}}{32 \pi^{3} \beta^{2}}\left(\frac{1}{|\mathbf{x}-\mathbf{a}|^{2}}-\frac{1}{|\mathbf{a}|^{2}}\right) .
$$

Since we are only interested in estimating the lowest-order modification of the static potential due to the presence of the Born-Infeld term, we have dropped a logarithmic factor in expression (24) which does not contribute in the limit $\beta \gg \theta$.

Accordingly, the potential for two opposite charges $e$ and $-e$ located at $\mathbf{y}$ and $\mathbf{y}^{\prime}$ is then given by

$$
V=\frac{e^{2}}{\pi} \ln \left(\theta\left|\mathbf{y}-\mathbf{y}^{\prime}\right|\right)-\frac{e^{4}}{16 \pi^{3} \beta^{2}} \frac{1}{\left|\mathbf{y}-\mathbf{y}^{\prime}\right|^{2}}
$$

in the short distance regime. It is interesting to note that this is exactly the result obtained for $Q E D_{3}$ with a Thirring interaction term among fermions, the so-called generalized Maxwell-Chern-Simons gauge theory $[13,16]$. Thus, from a physical point of view, one is led to the conclusion that the effect of including the Born-Infeld term is to generate stable bound states of quark-antiquark pairs at short distances, exactly as it happens with the Thirring term in the generalized Maxwell-Chern-Simons theory. In summary, the above analysis reveals that, although both theories are different, the physical content is identical in the short distance regime. 


\section{ACKNOWLEDGMENTS}

I would like to thank G. Cvetic for helpful comments on the manuscript. I would also like to thank I. Schmidt for his support. 


\section{REFERENCES}

[1] S. Deser, R. Jackiw, and S. Templeton, Ann. Phys. (N.Y.) 140, 372 (1982).

[2] G. Dunne, hep-th/9902115.

[3] A. Khare, Fractional Statistics and Quantum Theory (World Scientific, Singapore, 1998).

[4] T. Appelquist and R. D. Pisarski, Phys. Rev. D23, 2305 (1981).

[5] R. Jackiw and S. Templeton, Phys. Rev. D23, 2291 (1981).

[6] M. Stone, The Quantum Hall Effect (World Scientific, Singapore, 1992).

[7] A. A. Tseytlin, Nucl. Phys. B469, 51 (1996).

[8] G. W. Gibbons, hep-th/0106059.

[9] M. Born and L. Infeld, Proc. R. Soc. London A144, 425 (1934).

[10] P. K. Townsend, K. Pilch and P. van Nieuwenhizen, Phys. Lett. B136, 38 (1989).

[11] S. Deser and R. Jackiwv, Phys. Lett. B139, 2366 (1984).

[12] P. K. Tripathy and A. Khare, Phys. Lett. B504, 152 (2001).

[13] P. Gaete and I. Schmidt, Phys. Rev. D64, 027702 (2001)

[14] P. Gaete, Z. Phys. C76, 355 (1997).

[15] P. A. M. Dirac, The Principles of Quantum Mechanics (Oxford University Press, Oxford, 1958); Can. J. Phys. 33, 650 (1955).

[16] S. Ghosh, J. Phys. A33, 1915 (2000). 